\title{
Nanoparticles Self-Assembly Driven by High Affinity Repeat Protein Pairing
}

Kargal L. Gurunatha ${ }^{1}$, Agathe C. Fournier ${ }^{1}$, Agathe Urvoas², Marie Valerio-Lepiniec ${ }^{2}$, Valérie Marchi, ${ }^{3}$ Philippe Minard ${ }^{*}$ and Erik Dujardin ${ }^{1} *$

1 Groupe NanoSciences - CEMES-CNRS UPR 8011 - 29 rue J. Marvig, B.P. 94347, F-31055 Toulouse, France. Fax: (+33) 56225 7999. E-mail: dujardin@cemes.fr

2 I2BC, Univ Paris Sud, CNRS, CEA UMR 9198 - Bât. 430, F-91405 Orsay, France. Fax: (+33) 169853715. E-mail: philippe.minard@u-psud.fr

3 University Rennes 1, Institut of Chemical Sciences, UMR 6226 CNRS, Campus Beaulieu - F- 35042 Rennes Cedex France

\section{Supplementary Information}

3. Gel electrophoresis of constituent and assembled protein-functionalized nanoparticles at low colloidal concentration p. 6

4. Spectral shift of the plasmon resonance with the interparticle distance p. 7

5. Characterization of freestanding particle film assembled by protein pairing p. 9

6. Link between protein : nanoparticle stoichiometry and self-assembly topology p. 12 


\section{Materials}

Ultra-pure water with a conductivity of $18.2 \mathrm{M} \Omega / \mathrm{cm}$ was used throughout the experiments. Tetrachloroauric acid $\left(\mathrm{HAuCl}_{4}\right)$, trisodium citrate $\left(\mathrm{Na}_{3} \mathrm{C}_{6} \mathrm{H}_{5} \mathrm{O}_{7}\right)$, dithiothreitol $\left(\mathrm{C}_{4} \mathrm{H}_{10} \mathrm{O}_{2} \mathrm{~S}_{2}\right.$ - DTT), sodium phosphotungstate dibasic hydrate $\left(2 \mathrm{Na}_{2} \mathrm{O}_{2} \mathrm{P}_{2} \mathrm{O}_{5} 12 \mathrm{WO}_{3} \cdot 18 \mathrm{H}_{2} \mathrm{O}\right)$, boric acid $\left(\mathrm{H}_{3} \mathrm{BO}_{3}\right)$, sodium chloride $(\mathrm{NaCl})$ and agarose powder, sodium phosphate $(\mathrm{NaP}) 50 \mathrm{mM} \mathrm{pH} 7$ and $\mathrm{pH} 8$ buffers were purchased from Sigma-Aldrich and used as received. Negatively charged peptide, Cys-Cys-Cys-21-amino4,7,10,13,16,19-hexaoxaheneicosanoyl-Asp (C3E6Asp) (>90\% purity) was bought from Polypeptide Group and used as received. All glassware was cleaned with aqua regia $\left(\mathrm{HCl}: \mathrm{HNO}_{3}\right.$ 3:1) and rinsed abundantly with ultra-pure water. 


\section{TEM and UV visible absorption spectrum of citrate-stabilized Au nanoparticles.}

The citrate-stabilized Au nanoparticles were synthesized as described in Section 1 of this document and characterized by TEM and UV-visible spectrophotometry as described in Figure S1. The typical average particle diameter was $11 \mathrm{~nm}$ with a size distribution of $10-15 \%$. The nanoparticle exhibited a localized plasmon resonance at $520 \mathrm{~nm}$.
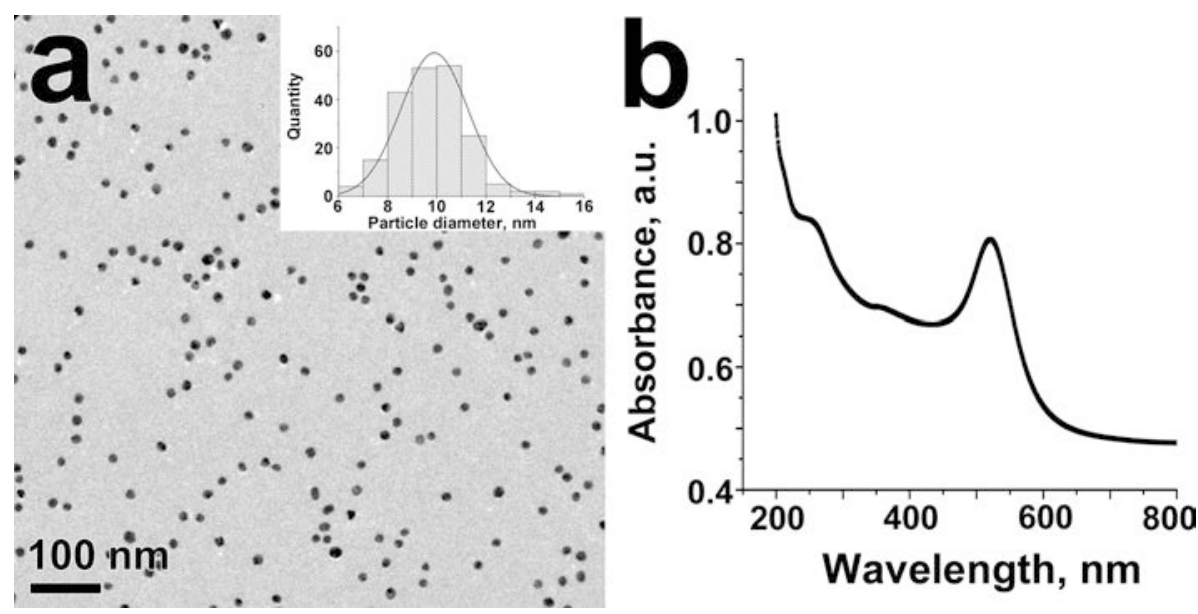

Figure S1. (a) TEM image of citrate-stabilized Au nanoparticles. They are stable and well separated with typical average diameter of 10-11 nm and a standard deviation of $10-15 \%$ as illustrated in the inset. (b) UV-visible extinction spectrum. 


\section{Gel electrophoresis of constituent and assembled protein-functionalized nanoparticles at low colloidal concentration}

The protein-driven self-assembly of nanoparticles results from the pairing equilibrium which is affected by the activity of the proteins in solution. As the proteins are immobilized on the surface of the nanoparticles, this activity depends on the nanoparticle concentration.

In Figure S2, the successive synthetic steps leading to protein-functionalized nanoparticles are reproduced by following the protocol described in the main text (Tracks 1 to 5 in Fig. 5). In tracks 6 and 7 , the particle suspensions are mixed at low nominal nanoparticle concentration, i.e. without the $5 x$ concentration step described at the end of the protocol.

It can be noticed that the self-assembly does proceed albeit to a much lesser extent, which is observable by the dilute and spread signal in tracks 6 and 7 .

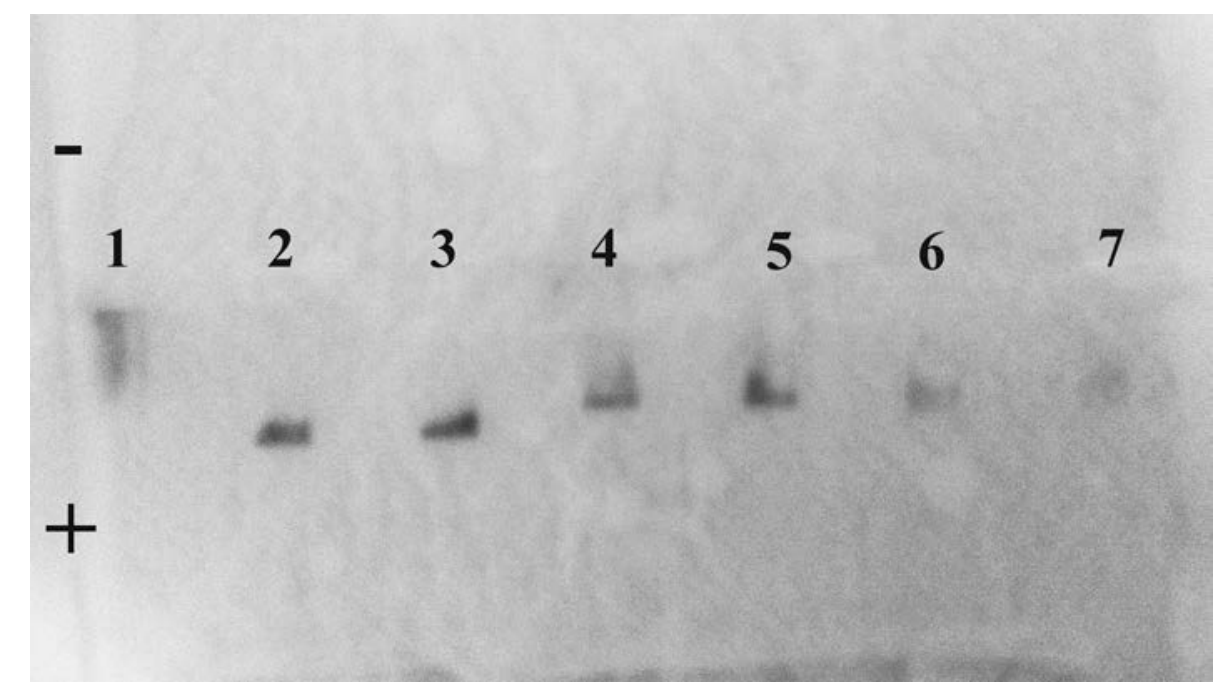

Figure S2. Migration of gold nanoparticle in agarose gel electrophoresis at low nanoparticle concentration. The protocol is identical with the one described in the main text except that the particle suspensions are not concentrated 5 times. Au NPs stabilized by (1) citrate or (2) C3E6Asp peptide. Tracks (3) to (5) correspond to Au NPs functionalized with $\alpha$-Rep proteins $A 3, \alpha 2$ and $\alpha 17$ respectively. The conjugates $\mathrm{Au}-\mathrm{A} 3 \bullet \alpha 2-\mathrm{Au}$ and $\mathrm{Au}-\mathrm{A} 3 \bullet \alpha 17-\mathrm{Au}$, prepared without the $5 \mathrm{x}$ concentration step, are run in tracks (6) and (7) respectively. 


\section{Spectral shift of the plasmon resonance with the interparticle distance}

When Au nanoparticles are brought very close to each other, the localized surface plasmon band (LSP) undergoes a spectral shift towards higher wavelengths because of the dipolar coupling of the LSP borne by the nearest neighbors. ${ }^{1}$ This has been widely documented in aggregates of nanoparticles obtained by direct coagulation or by self-assembly driven by small molecules. When, the interparticle gap distance is as small as $1 \mathrm{~nm}$, the spectral shift can be as large as $200 \mathrm{~nm}^{1,2}$

However, this spectral shift rapidly vanishes as the gap distances increases.

In Figure $\mathrm{S} 3 \mathrm{a}$, the absorption spectra of $11-\mathrm{nm} \mathrm{Au}$ nanoparticle dimers placed in a medium of refractive index $n=1.5$ are calculated as a function of the gap distance of 3,5 and $7 \mathrm{~nm}$ and compared to the one of isolated nanoparticles. A gap distance of $3 \mathrm{~nm}$ does produce a distinct redshift but for a gap of more than $5 \mathrm{~nm}$, this shift is almost imperceptible.

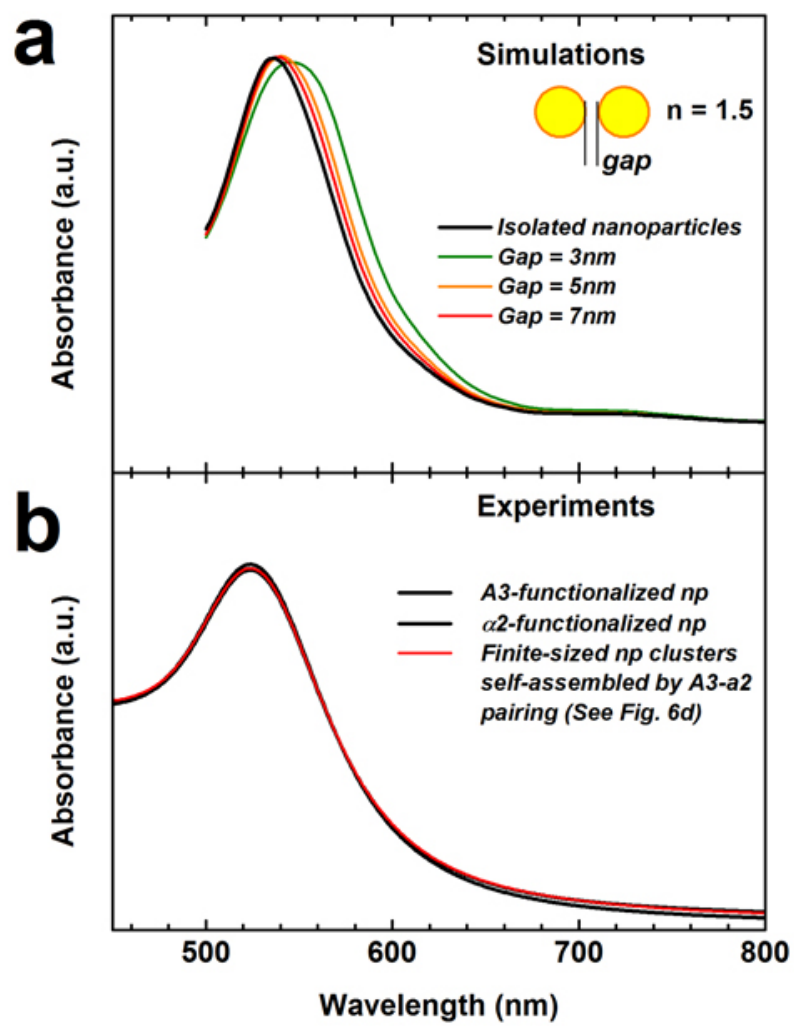

Figure S3. (a) Evolution of the absorption spectra of 11-nm Au nanoparticle dimers as a function of the interparticle gap distance. The refractive index of the surrounding medium is 1.5 (glass). (b) Absorption spectra of isolated nanoparticles functionalized with $\mathrm{A} 3$ or a2 proteins (black) and of the self-assembled finite size clusters shown in Fig. 6d (red).

This idealized situation can be compared to the spectra of isolated Au nanoparticles functionalized with complementary proteins (A3, $\alpha 2$ or $\alpha 17)$ as described in the main text and the spectra of the selfassembled structure. Figures $6 \mathrm{~d}$ and $6 \mathrm{e}$ present the spectra for the high protein : nanoparticle molar ratio $(30: 1)$ leading to monolayered films and Fig. S3b shows, for the $A 3 \bullet \alpha 2$ system, the spectra for the low protein : nanoparticle molar ratio $(20: 1)$ that is shown to yield finite sized nanoparticle clusters (Fig .6c, d). We observe that the spectral shift upon assembly is imperceptible.

The main reason in the case of the monolayered film (Fig. $5 \mathrm{~d}, \mathrm{e}$ ) is that the film settles (or has a small absorption cross-section) and is not probed by spectrophotometry. The spectra (6) and (7) shown in Fig5d-e are due to the nanoparticle NOT assembled in the film but still individually suspended, which thus exhibit a non-shifted maximum. The formation of the film is nevertheless revealed in those 
spectra by the decrease in absorption corresponding to the nanoparticles immobilized in the selfassembled film.

For the clusters, which remain in suspension, the absence of shift is consistent with the average gap size that exceeds $5.5 \mathrm{~nm}$ (See Section 5 and Fig. S6). We should point out that the simulations in Fig. S3a are done in a glass-like medium of refractive index $n=1.5$ in order to increase the redshift, which consequently also redshift the LSP maximum $\left(\lambda_{\max } \sim 535 \mathrm{~nm}\right)$ compared to the aqueous medium of index $n=1.33$ used in the experiments of Fig. S3b $\left(\lambda_{\max }{ }^{`} 520 \mathrm{~nm}\right)$. Moreover, the simulated LSP bands are narrower than the experimental ones because the ohmic losses are slightly underestimated. Consequently, the experimental redshift for a gap size equal or greater than $5 \mathrm{~nm}$ is expected to be smaller than the ones obtained in the simulations. Both simulations and experiments are therefore consistent with the fact that protein-pair driven self-assembly, as designed in this work, does not lead to a measurable spectral shift of the LSP. 


\section{Characterization of freestanding particle film assembled by protein pairing}

Web-like filaments that spontaneously form within 48 hours after mixing A3-Au nanoparticles with $\alpha 2-$ Au or a17-Au nanoparticles were analyzed by SEM (Fig. S4) and TEM (Fig. S5). For this, droplets containing the filaments were deposited onto a chip of oxidized silicon wafer and left to dry.

Figure S4 shows SEM images taken at several magnifications. Fig. S4a shows that the filaments are made of a uniform, one-particle-thick film that extends overs tens of micrometers. Zoomed in views reveal that the nanoparticles are densely but uniformly packed but no sign of three dimensional stacking or clustering is observed (Fig. S4b). Very occasionally, a second partial monolayer can be observed as in Fig. S4c but it was not possible to attribute such bilayers to self-folded monolayer areas or to areas where self-assembly produced a bilayer region.

The variation in compaction observed in Fig. S4d-f can be attributed to the variation of protein pair crosslinking as the effective concentration increased inside the drying droplet. The structure of the monolayered film in the aqueous medium would thus be similar to the dark areas in Fig. S4d also depicted in Fig. S4f.

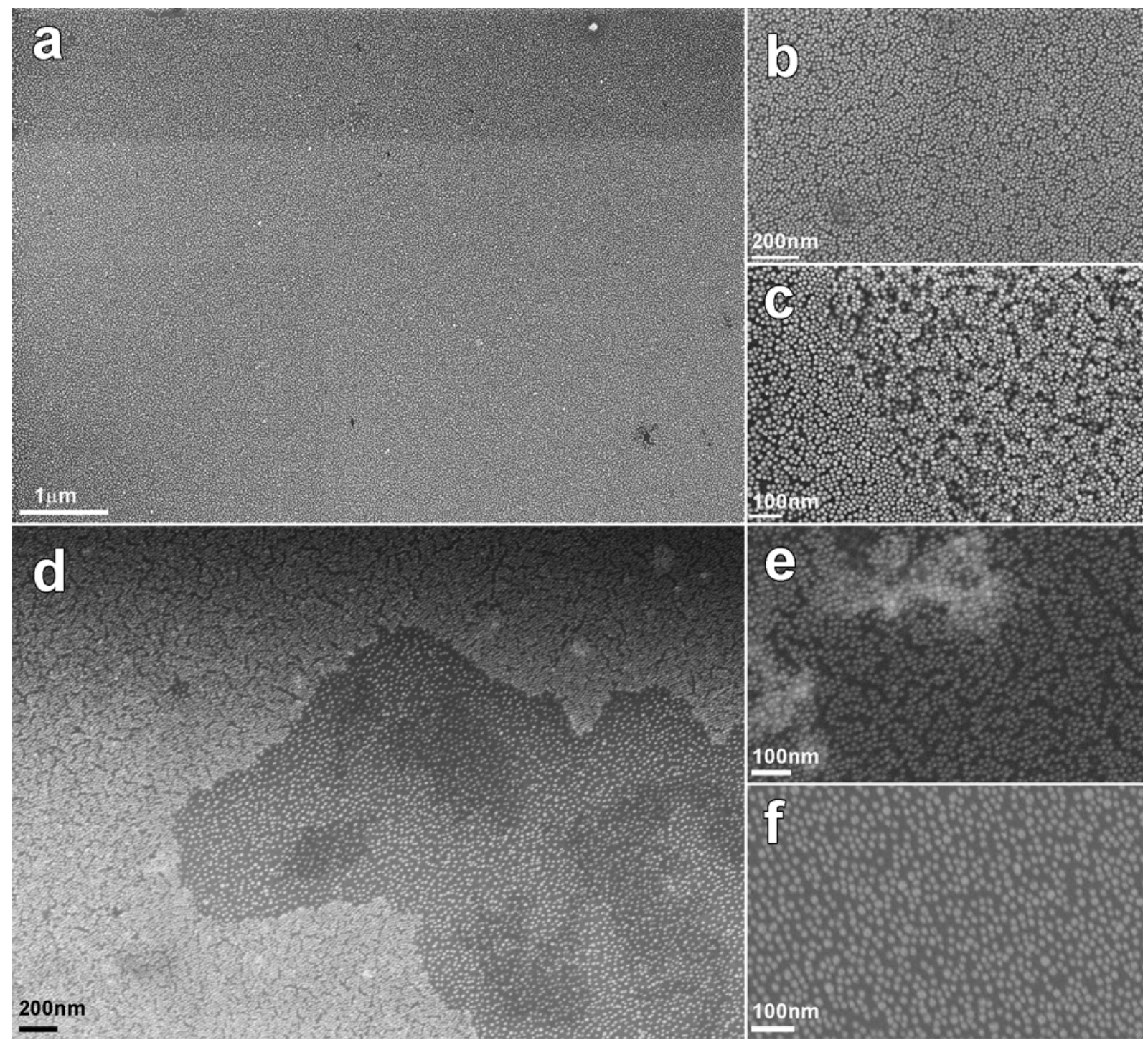

Figure S4. SEM image of Au-A3 conjugate mixed with Au-A17 protein conjugate. (a) Low magnification view of the self-assembled monolayer of Au nanoparticles. (b) Closer view of the monolayer showing the compact arrangement of nanoparticles. (c) Portion of bi-layer found in some rare places. (d) Low magnification view of an area of the monolayer with two levels of compaction. The bright close packed area is magnified in (e) and the more loosely packed zone is magnified in (f). 
In complement to Fig. 1 and Fig. $6 a$ in the main text, Figure S5 displays TEM images of free-standing film at low (a-c) and high (d) magnifications. The highly homogeneous monolayered film (including along its edges), the absence of lone clusters and the very few individual nanoparticles in the vicinity of the film edge confirms that the observed film is produced in suspension and not by drying/dewetting on the supporting carbon film. The nanoparticles are surrounded by organic matter with a typical distance to nearest neighbors of about $3-5 \mathrm{~nm}$ as seen in Fig. S5d, which is similar to the SEM observations in Fig. S4f.

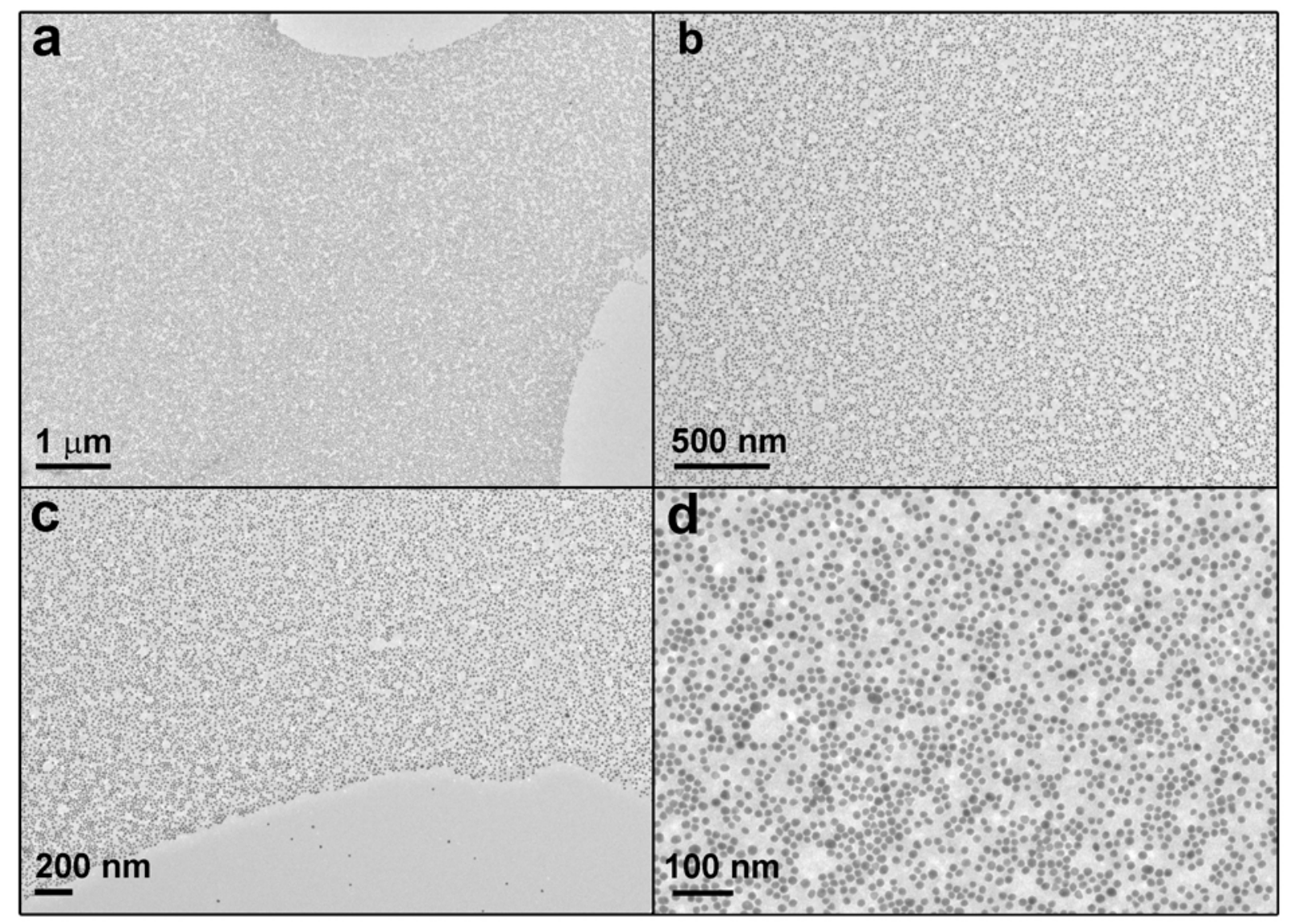

Figure S5. TEM images of Au-A3 conjugate mixed with Au-A17 protein conjugate. $(a, b, c)$ Low magnification views of the self-assembled monolayered film of Au nanoparticles. (d) Closer view of the monolayer showing the compact arrangement of nanoparticles surrounded by organic material.

Within the free standing films constituting the web-like filaments, the Au nanoparticles could be seen as densely and regularly packed with a uniform finite interparticle distance. Figure S6 shows two histograms of the interparticle distance measured from TEM and SEM images. Both distributions coincide on a mean distance of about $3.5 \pm 1.2 \mathrm{~nm}$ and $4.3 \pm 0.7 \mathrm{~nm}$ that is in good agreement with the spherical volume of the protein pair of about $5 \mathrm{~nm}$ diameter.

A more detailed analysis of SEM and TEM images such as Fig. S4b or Fig. S5b is provided by the 2D auto-correlation analysis of the SEM image in Fig. S6c, shown in Fig. S6d. The auto-correlation shows clear maxima on close-packed sites with intensity maxima along two or three hexagonal rings that indicate a significant correlation with first, second and even third nearest neighbors. The average center-to-center interparticle distance is $17.5 \mathrm{~nm}$, which corresponds to an interparticle gap of about $5.5 \mathrm{~nm}$. 

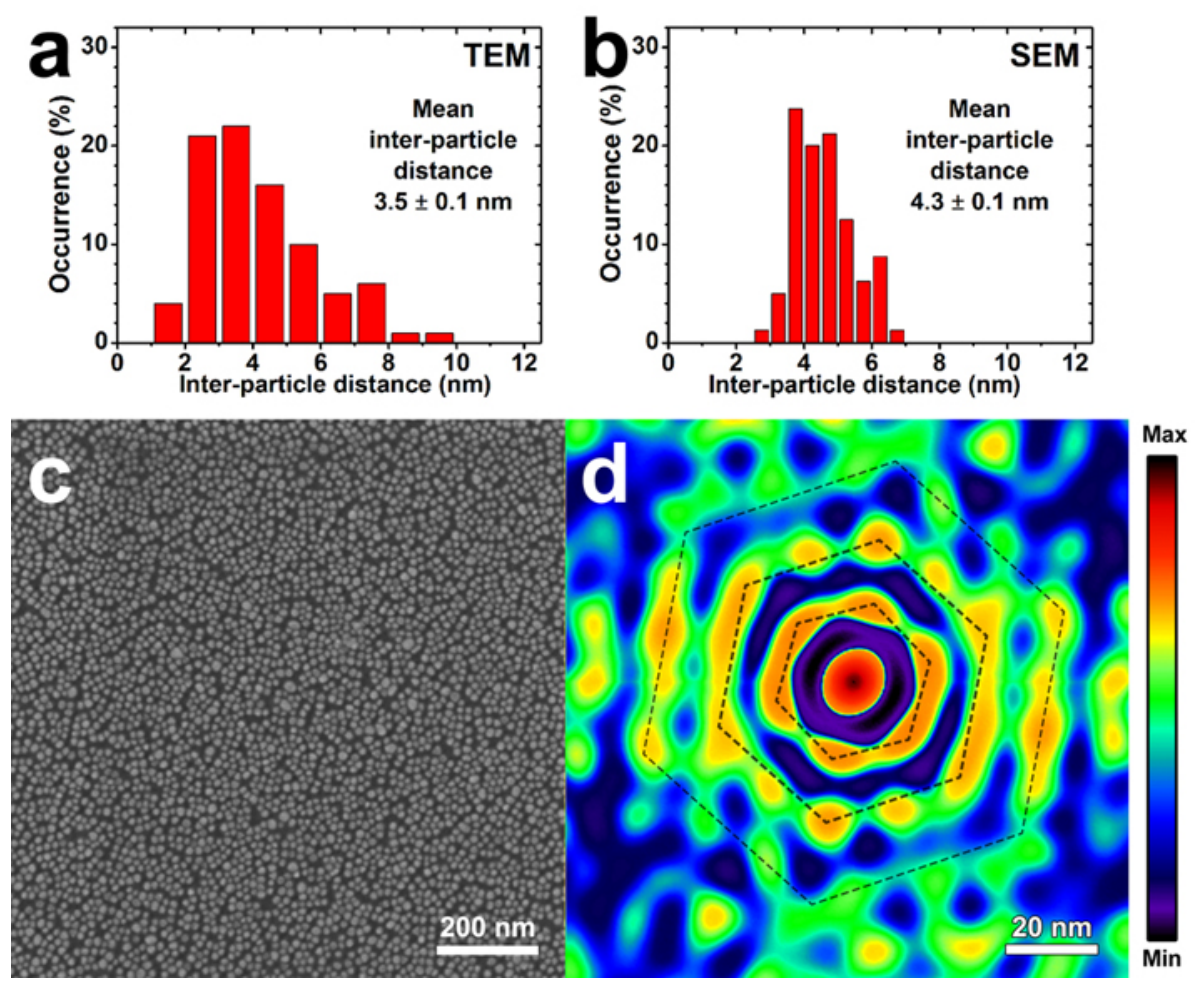

Figure S6. $(a, b)$ Histograms of interparticle distances measured on (a) TEM and (b) SEM images of the nanoparticle filamentous film collected after mixing Au-A3 and Au- $\alpha 17$ conjugates. (c) SEM image and (d) corresponding 2D auto-correlation image showing short range close packed order with first and second nearest neighbors clearly identified by maxima in the auto-correlation function. The third nearest neighbor corona is partially visible too. The center-to-center distance is $17.5 \mathrm{~nm}$ corresponding to an interparticle distance of about $5.5 \mathrm{~nm}$.

We note that free-standing nanoparticle films with thickness as small as one nanoparticle have been reported on multiple occasions in the past decade,[8-13] which indicates that 2D assembly is not as unfavorable as it could first appear. While no general mechanism accounts for all systems, which all have specific reasons to undergo 2D assembly, one can distinguish two types of rationale. Nanoparticles with isotropic ability to self-assemble (for example uniformly coated with complementary DNA strands) are confined in 2D spaces by spinning, surface templating, dewetting across small apertures, etc. Alternatively nanoparticles with regioselective and directional assembling interactions can form 2D films in solutions. Anisotropic dipolar interactions, coplanar interacting patches have been shown to yield free-standing films.

In our case, the $\alpha$-Rep pair formation implies that the concave facet is freely accessible and therefore the proteins (in particular A3) are probably positioned radially to the nanoparticle surface thanks to the $\mathrm{Cys}_{3}$ linker placed at the C-terminal.

Moreover, owing to their modular structure, the proteins bear a well-defined distribution of charges in the non-variable part of the proteins. In particular, two longitudinal ridges of negative charges are present in the loops bridging the successive double $\alpha$-helices (sequences DED and DER before and after each helix). When one takes into account the fact that $A 3$ and $\alpha 17$ are rather large ( $5 \mathrm{~nm}$ long) compare to the nanoparticles size $(10 \mathrm{~nm})$, it is therefore possible that 3 proteins attached to a given nanoparticle adopt a regular equatorial arrangement.

The rigid protein pair formation evidenced by XRD in our earlier work would preserve the directionality of particle-to-particle attachment through the protein pair.[14] Although this work on the self-assembly of particles decorated with flat interacting patches, which departs from our case where the nanoparticles and the proteins are of similar size, it has shown that nanoparticles with selective directional interactions from equatorial patches leads to stable film formation. Moreover when the 
particle bear only two patches with a relative azimuthal angle smaller than $180^{\circ}$, particle rings are formed which are indeed observed in our films (Fig. S5d).

We therefore believe that the combination of low stoichiometry, regular equatorial surface layout of the proteins due to the inter-protein charge interactions and steric hindrance along with rigid protein pair formation contribute to favor coplanar assembly of the nanoparticles. 


\section{Link between protein : nanoparticle stoichiometry and self-assembly topology}

The grafting of the a-Rep proteins onto the nanoparticles is obtained through thiol-Au bond formation, which is quantitative, the surface chemistry is not limiting and more than one protein can definitely be attached to the nanoparticles. Yet their maximum number is rather limited owing the comparable size of the proteins ( $\mathrm{A} 3$, and $\alpha 17)$ with the nanoparticles and the significant charge borne by them at $\mathrm{pH} 7$. If the nanoparticles were covered with more than 4 proteins, reticulated and isotropic 3-dimensional aggregation would occur which was not seen for the finite sized aggregates nor alongside the extended film formation. This observation indicates that the stoichiometry remains essentially between 1:1 and 3:1 protein per particle.

In Figure 5, the breadth of the GEP bands No3 and 5 indicates that we have a range of stoichiometry for the high feeding ratio. The corresponding GEP bands No3 and 5 obtained for the low feeding ratio (Fig. S2) are both narrower and relatively closer to the injection well. This observation is consistent with a smaller number of proteins attached, on average, on the nanoparticles and the stated observation that a higher feeding ratio leads to larger aggregates (film) and a smaller feeding ratio to finite sized clusters. A similar interpretation of $\alpha 2$ is less straightforward since this protein is much smaller and the variation of stoichiometry does not affect significantly the charge / size of the bioconjugate.

When nanoparticles bear 1, 2, 3 or more proteins able to bind complementary partners, the topology of the resulting self-assembly structure will depend on the relative amount of each types of nanoparticles, as illustrated in Figure S7.
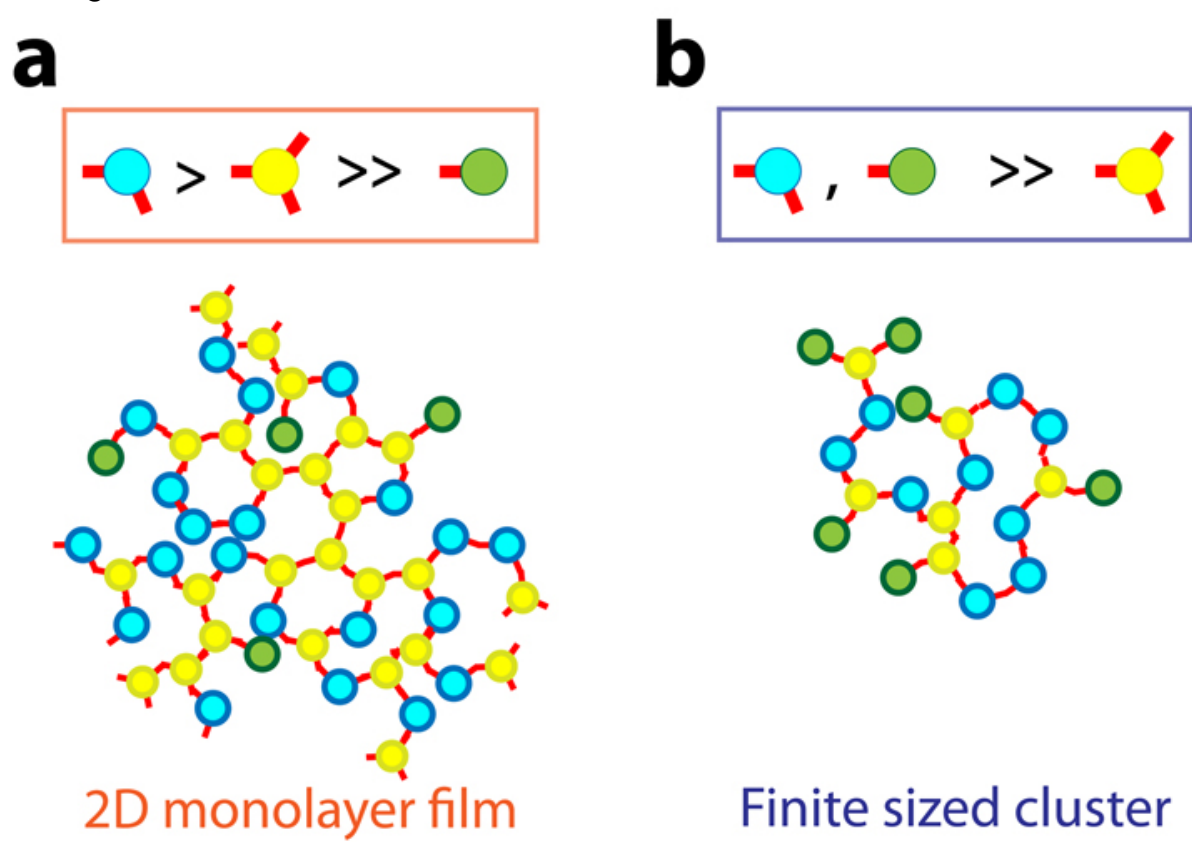

Figure S7 Schematics of some topologies adopted by self-assembled nanoparticles superstructures when relative amount of particles bearing 1, 2 or 3 binding proteins is modified. (a) When nanoparticles bearing a single proteins are much less numerous than ones bearing 2 or 3 proteins, the network can expand largely. If ditopic particle prevail, the 3-dimnesional branching is limited and the resulting self-assembly can be restricted to monolayered films. (b) If particles bearing 3 proteins are present but in small amount, the di- and monotopic particles will rapidly limit the growth extension and finite sized clusters are formed.

With pure 1:1 stoichiometry, i.e. nanoparticles bearing a single protein, only dimeric structure can be produced. 
With a mixture or mono- and ditopic (2:1 stoichiometry) particles, chains will be formed, the length of which will vary with the relative amount of mono- vs ditopic particles.

As soon as tri-topic particles (3:1 stoichiometry) are introduced, the self-assembled structures can expand as a 3D network, which will grow all the more that monotopic particles are scarce and unable to stop the nanoparticle concatenation. Yet, the relative amount or di- vs tritopic particles may favor the formation of 2D assembly. In particular if tri-topic nanoparticles are significantly less frequent than ditopic ones (Fig. S7a), the potential for 3D networking is limited and the self-assembly can proceed in 2D resulting in monolayered film as observed in this work and earlier reports for semiconductive nanoparticles (Kotov et al., Science 2006, 314, 274-278) and for DNA-linked metal nanoparticles (D. Luo et al, Nature Materials, 2009, $\underline{8}$, 519-25), for example.

If the number of available proteins is further reduced, leading to few tri-topic particles but mostly diand monotopic particles, the expansion of the self-assembly is limited by the incorporation of monotopic particles that stop the chain growth, thus quickly stopping the development of the aggregates. This results in finite sized clusters as illustrated in Fig. S7b and experimentally observed in our case in Figs. $6 \mathrm{c}$ and $6 \mathrm{~d}$. 


\section{References}

1. Dujardin, E.; Girard, C. Plasmonic Nanoparticle Networks. In Handbook of Nanophysics Sattler, K., Ed. Taylor \& Francis: London, 2010; Vol. 3.

2. Lin, S.; Li, M.; Dujardin, E.; Girard, C.; Mann, S. One-Dimensional Plasmon Coupling by Facile Self-Assembly of Gold Nanoparticles into Branched Chain Networks. Adv. Mater. 2005, 17, 25532559. 